Second Meeting, 9th December 1904.

Mr W. L. Thomson, President, in the Chair.

\title{
The Proof by Projection of the Addition Theorem in Trigonometry.
}

By D. K. Picken, M.A.

The object of this paper is to remove the difficulty that arises in giving a general proof by projection methods of this theorem, without in any way interfering with the single-valuedness of the position of a radius vector tracing out angles from a given initial position, when the values of the trigonometrical ratios are given.

It is necessary, first of all, to give a clear statement of the definitions and theorems in Projection.

Definition: The projection of a point $S$ on a straight line $\mathrm{XY}$ is the foot $Z$ of the perpendicular from $S$ on $X Y$.

As the point $S$ moves in any manner, the point $Z$ moves backwards and forwards along $X Y$. If we call the amount of a motion of $\mathrm{Z}$ from $\mathrm{X}$ towards $\mathrm{Y}$ a positive segment, and that of a motion in the opposite direction a negative segment, the total displacement of $Z$ corresponding to a given motion of $\mathbf{S}$ is a positive or a negative segment, which is the algebraic sum of the alternately positive and negative segments which $Z$ describes during the motion. Also, if $S$ is given a succession of motions, the total displacement of $Z$ is the algebraic sum of the displacements due to the several motions (vide Theorem IV. infra.).

Definition: If $\mathbf{S}$ moves along a straight line $\mathrm{PQ}$ from $\mathrm{P}$ to $\mathrm{Q}$, the positive or negative segment $M N$ described by $Z$ is called the projection of $\mathrm{PQ}$ on $\mathrm{XY}$.

The following theorems are then obvious:-

I. The projection of $\mathrm{QP}$ on $\mathrm{XY}=-$ (the projection of $\mathrm{PQ}$ on $\mathrm{XY}$ ).

II. If $U V$ is equal to, parallel to and in the same direction as $P Q$ the projection of $U V$ on $X Y=$ the projection of $P Q$ on $X Y$. 
III. If $\mathrm{R}$ be any point of the unlimited line through $\mathrm{P}$ and $\mathrm{Q}$, so that $\mathrm{PR}=n . \mathrm{PQ}$ where $n$ is any real number, the projection of $\mathrm{PR}$ on $\mathrm{XY}=n$ (the projection of $\mathrm{PQ}$ on $\mathrm{XY}$ ); for the projection $K$ of $R$ lies between $M$ and $N$, on $M N$ produced, or on NM produced, according as $\mathrm{R}$ lies between $\mathbf{P}$ and $\mathrm{Q}$, on $\mathrm{PQ}$ produced, or on $\mathrm{QP}$ produced.

IV. If $P$ and $Q$ be joined by a succession of straight lines

$$
P Q_{1}, Q_{1} Q_{2}, \ldots Q_{r-1} Q
$$

the projection of $P Q$ on $X Y$

$=$ the sum of the projections of $P Q_{1}, Q_{1} Q_{2}, \ldots Q_{r-1} Q$ on $X Y$.

The generality of the proof given below of the Addition Theorem depends on Theorem III.

The Trigonometrical Ratios.

Let angles $\theta$ be described by the turning in one plane of a straight line $O P$ about a fixed point $O$ in it, from a fixed initial position OA. The words positive and negative can then obviously be applied to distinguish the two kinds of turning. Let $O B$ be the position of $\mathrm{OP}$ when $\theta$ is a positive right angle, and let $\mathrm{AO}, \mathrm{BO}$ produced meet the circle described by $P$ in $A^{\prime}$ and $B^{\prime}$.

\section{Definitions :}

The ratio (projection of $O P$ on $B^{\prime} O B$ : length of $O P$ ) is called the sine of $\theta$.

The ratio (projection of $O P$ on $A^{\prime} O A$ : length of $O P$ ) is called the cosine of $\theta$; etc., etc.

It follows that these trigonometrical ratios are single-valued functions of the position of the vector $O P$, and that when $\sin \theta$ and $\cos \theta$ are given the position of $O P$ is uniquely defined.

If $O Q, O Q^{\prime}, O Q^{\prime \prime}$ are the positions of $O P$ when $\theta=a,-\alpha$ and $\left(a+\frac{\pi}{2}\right)$, it is easy to obtain from consideration of the relative positions of $Q, Q^{\prime}, Q^{\prime \prime}$ on the circle, general proofs of the formulae:

$$
\begin{aligned}
& \sin (-\alpha)=-\sin \alpha ; \cos (-\alpha)=\cos \alpha \\
& \sin \left(\alpha+\frac{\pi}{2}\right)=\cos \alpha ; \cos \left(\alpha+\frac{\pi}{2}\right)=-\sin \alpha .
\end{aligned}
$$


The Addition Theorem. (Fig. 27.)

Let $O A_{1}$ be the position of $O P$ when $\theta=a, O B_{1}$ when $\theta=a+\frac{\pi}{2}$; and let angles $\phi$ be measured by the turning of OP from the initial position $O A_{1}$. Let $O Q$ be the position of $O P$ when $\phi=\beta$; then $O Q$ is the position of $O P$ when $\theta=\alpha+\beta$. Let $M_{1}, N_{1}$ be the projections of $Q$ on $A_{1}{ }^{\prime} O A_{1}$ and $B_{1}{ }^{\prime} O B_{1}$.

We have then

$O Q \cos (a+\beta)$

$=$ projection of $O Q$ on $A^{\prime} O A$

$=\left(\right.$ projection of $O M_{1}+$ projection of $\left.M_{1} Q\right)$ on $A^{\prime} O A$ [Thm. IV.]

$=\left(\right.$ projection of $\mathrm{OM}_{2}+$ projection of $\left.\mathrm{ON}_{1}\right)$ on $\mathrm{A}^{\prime} \mathrm{OA}$ [Thm. II.]

$=\left\{\right.$ projection of $\left(\cos \beta . O A_{1}\right)+$ projection of $\left.\left(\sin \beta . O B_{1}\right)\right\}$ on $A^{\prime} O A$

$=\left\{\cos \beta\left(\right.\right.$ projection of $\left.O A_{1}\right)+\sin \beta\left(\right.$ projection of $\left.\left.O B_{1}\right)\right\}$ on $A^{\prime} O A$

$=\cos \beta \cdot\left(\mathrm{OA}_{1} \cos \alpha\right)+\sin \beta\left\{\mathrm{OB}_{1} \cos \left(a+\frac{\pi}{2}\right)\right\} ;$

[Thm. III.]

$$
\begin{aligned}
\therefore \quad \cos (\alpha+\beta) & =\cos \alpha \cos \beta+\cos \left(\alpha+\frac{\pi}{2}\right) \sin \beta \\
& =\cos \alpha \cos \beta-\sin \alpha \sin \beta .
\end{aligned}
$$

Similarly, by projecting on $\mathrm{B}^{\prime} \mathrm{OB}$, we get

$$
\begin{aligned}
\sin (\alpha+\beta) & =\sin \alpha \cos \beta+\sin \left(\alpha+\frac{\pi}{2}\right) \sin \beta \\
& =\sin \alpha \cos \beta+\cos \alpha \sin \beta
\end{aligned}
$$

and the theorems are true whatever be the sign and whatever the magnitude of the angles $\alpha$ and $\beta$. 